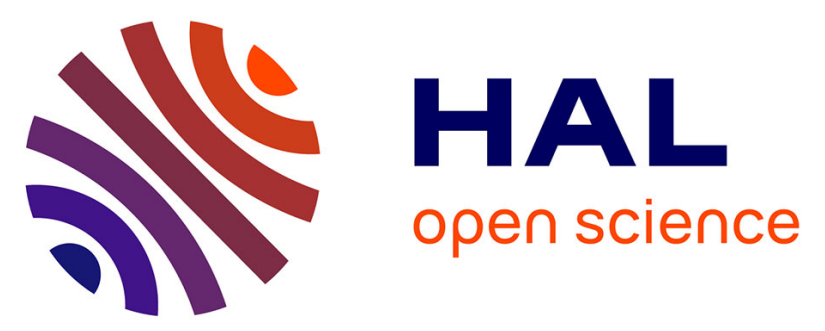

\title{
Utilisation des dynamomètres isocinétiques pour la caractérisation des propriétés mécaniques passives du système musculo-articulaire
}

\author{
Giuseppe Rabita, Pierre Portero, Stevy Farcy
}

\section{To cite this version:}

Giuseppe Rabita, Pierre Portero, Stevy Farcy. Utilisation des dynamomètres isocinétiques pour la caractérisation des propriétés mécaniques passives du système musculo-articulaire. Movement \& Sport Sciences - Science \& Motricité, 2014, 85, pp.103-108. 10.1051/sm/2013091 . hal-01702776

\section{HAL Id: hal-01702776 https://hal-insep.archives-ouvertes.fr/hal-01702776}

Submitted on 7 Feb 2018

HAL is a multi-disciplinary open access archive for the deposit and dissemination of scientific research documents, whether they are published or not. The documents may come from teaching and research institutions in France or abroad, or from public or private research centers.
L'archive ouverte pluridisciplinaire HAL, est destinée au dépôt et à la diffusion de documents scientifiques de niveau recherche, publiés ou non, émanant des établissements d'enseignement et de recherche français ou étrangers, des laboratoires publics ou privés. 


\title{
Utilisation des dynamomètres isocinétiques pour la caractérisation des propriétés mécaniques passives du système musculo-articulaire
}

\author{
Pierre Portero $^{1,2}$, Stevy Farcy ${ }^{1,3}$ et Giuseppe Rabita ${ }^{3}$ \\ 1 EAC CNRS 4396, Université Paris Est Créteil, Créteil, France \\ 2 Service de Rééducation Neuro-orthopédique, Hôpital Rothschild (AP-HP), 5 rue Santerre, 75571 Paris Cedex 12, France \\ 3 Service Recherche, Institut National du Sport, de l'Expertise et de la Performance, INSEP, Paris, France
}

Reçu le 8 juin 2013 - Accepté le 26 juin 2013

\begin{abstract}
Résumé. Les dynamomètres isocinétiques sont classiquement utilisés pour la mesure des variables caractéristiques du muscle (force, puissance, endurance, etc.), moins d'attention a été apportée aux possibilités qu'ils offrent de mesurer les propriétés passives du système musculoarticulaire (SMA). Ces systèmes sont configurés de façon i) à mobiliser passivement l'articulation à des vitesses faibles et sur la quasi-totalité de l'amplitude articulaire, ii) à maintenir un niveau constant d'étirement ou de contrainte, iii) à réaliser un protocole de charge-décharge, iv) à solliciter de façon cyclique le SMA. Le comportement mécanique du SMA peut être caractérisé par ses propriétés de raideur et viscoélasticité. D'autres méthodes ont permis, soit de contrôler les conditions passives de la méthode comme l'électromyographie de surface, soit d'accéder aux propriétés mécaniques du complexe muscle-tendon (CMT).
\end{abstract}

Mots clés : Isocinétisme, système musculo-articulaire, propriétés mécaniques passives, raideur, viscoélasticité

\begin{abstract}
Using isokinetic dynamometers for the characterisation of the passive mechanical properties of the musculoarticular system.

Traditionally, isokinetic dynamometers have been used to measure variables associated with strength, power and endurance characteristics of muscle. Much less attention has been given to their potential for measuring the passive mechanical properties of the musculoarticular system (MAS). These dynamometers are configured in order to i) mobilise passively the joint at low speeds and over all the range of motion, ii) maintain a steady level of stretching or stress, iii) realise a loading-unloading protocol, iv) apply cyclic stretching on the MAS. The mechanical behaviour of the MAS can be characterised by stiffness and viscoelasticity properties. Other methods allowed, either to control the passive conditions of the method as the surface electromyography, or to reach the mechanical properties of the muscle-tendon complex.
\end{abstract}

Key words: Isokinetics, musculoarticular system, passive mechanical properties, stiffness, viscoelasticity

\section{Introduction}

Les dynamomètres isocinétiques sont connus pour être des outils de référence pour la caractérisation des propriétés contractiles musculaires in vivo chez l'homme selon différents modes (Pincivero, Lephart, \& Karunakara, 1997 ; Taylor, Sanders, Howick, \& Stanley, 1991). Cependant leur utilisation pour la réalisation des mesures des propriétés passives est moins connue.
Cet article a pour objectif de décrire les méthodes les plus courantes pour évaluer l'extensibilité des tissus musculo-tendineux à l'aide de dynamomètres isocinétiques. Nous illustrerons ces méthodes à partir d'études issues de la littérature et fournirons des exemples de résultats qui montrent comment l'utilisation de l'isocinétisme dans ce domaine a permis d'améliorer la connaissance des facteurs altérant l'extensibilité du système musculo-articulaire (SMA). 


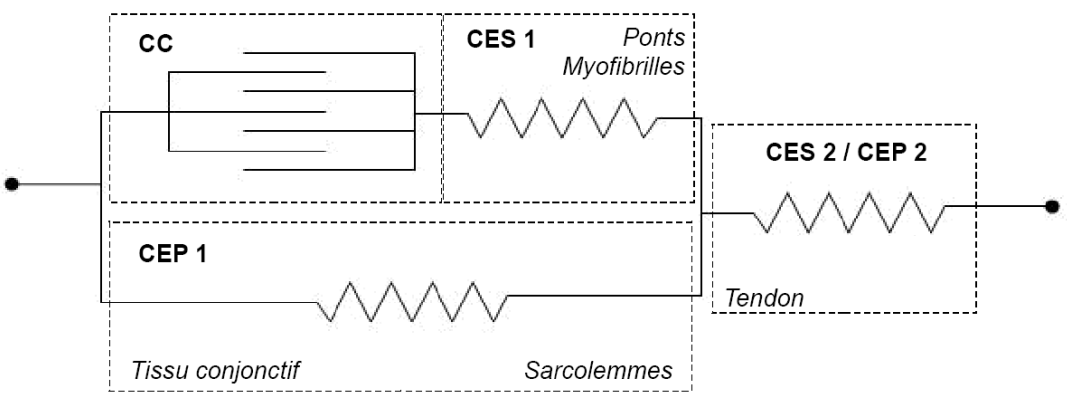

Fig. 1. Modèle de muscle proposé par Zajac (1989). CC : composante contractile; CES : composante élastique série; CEP : composante élastique parallèle.

\section{Bases biomécaniques}

La caractérisation des propriétés mécaniques du muscle fait en général appel à un modèle rhéologique de muscle initialement développé par Hill (1951). De nombreuses méthodes in vivo ont été adaptées à partir de ce modèle (Goubel \& Lensel-Corbeil, 2003). Ce modèle comprend classiquement une composante contractile (CC), une composante élastique série (CES) et une composante élastique parallèle (CEP). D'autres modèles de muscle, comme celui proposé par Zajac (1989), placent les structures tendineuses (CES2 ou CEP2) en série des CES1 et CEP1 (Fig. 1).

La CES est classiquement caractérisée lors de contractions musculaires, pour des longueurs de muscle n'induisant pas de tension musculaire passive, et a fait l'objet de nombreuses études (Farcy, Portero, \& Rabita, 2011). La contribution de la CEP à la production de force du complexe muscle-tendon (CMT) isolé est connue (voir Goubel \& Lensel-Corbeil, 2003). La CEP est également fortement impliquée dans la résistance à l'étirement produite par le SMA lors de la mobilisation passive d'une articulation (pour revues, voir Gajdosik, 2001; Magnusson, Aagard, Simonsen, \& Bojsen-Moller, 1998; McNair \& Portero, 2005). En situation écologique, la contribution de la CEP au couple articulaire lors de tâches telles que la marche ou la course est plus récente (Silder, Heiderscheit, \& Thelen, 2008). Cette composante passive présente donc un intérêt fonctionnel évident d'où la nécessité de la caractériser.

\section{Méthodes de caractérisation du comportement mécanique du SMA passif in vivo}

De nombreuses études ont utilisé ce type de dynamomètre pour mobiliser passivement une articulation tout en mesurant la résistance à l'étirement du SMA (Gajdosik, 2001; Magnusson, et al., 1998; McNair \& Portero, 2005 ; Nordez, Cornu, \& McNair, 2006 ; Nordez, Casari, \& Cornu, 2008) et à différentes vitesses (Gajdosik, et al., 2005; Lamontagne, Malouin, \& Richards, 1997; Rabita, et al., 2005; Engsberg, Olree, Ross, \& Park, 1996; Engsberg, Ross, Olree, \& Park, 2000; Damiano, et al., 2002 ; Ross \& Engsberg, 2002).
L'utilisation de ces dynamomètres isocinétiques pour l'exploration passive du SMA a nécessité d'étudier la sensibilité et la validité des mesures (Nordez, et al., 2008). En effet, au cours de mouvements passifs, le niveau des couples résistants est très inférieur à celui des couples générés lors de la contraction volontaire (e.g. extenseurs du genou : $300 \mathrm{Nm}$ en actif vs. 20-30 Nm en passif) (Magnusson, et al., 1998; Nordez, et al., 2008). De plus les variations observées, consécutives à différents protocoles (e.g. stretching) sont faibles $(<3 \mathrm{Nm}$ ) (Nordez, et al., 2008). Une faible variation ou une erreur de mesure considérée comme négligeable en mode actif ne l'est plus dans la quantification en mode passif.

\section{SMA étudiés}

Dans la littérature, les groupes musculaires les plus étudiés lors d'étirements passifs sont les ischios-jambiers et les fléchisseurs plantaires, et donc les systèmes isocinétiques sont configurés de façon à mobiliser passivement les articulations du genou et de la cheville. Pour le genou, la mise en tension passive des ischio-jambiers se fait soit en mobilisant le genou, la hanche étant bloquée en flexion (Magnusson, et al., 1998), soit en mobilisant la hanche, le genou étant bloqué en extension (Halbertsma, Mulder, Goeken, Gijeken, \& Eisma, 1999). Pour la cheville, le sujet est allongé (en décubitus dorsal ou ventral), la cheville positionnée entre 0 et 45 degrés de flexion plantaire, et le genou étendu. La mobilisation se fera en flexion dorsale afin d'étirer les fléchisseurs plantaires (Maïsetti, Sastre, Lecompte, \& Portero, 2007 ; McNair, Dombroski, Hewson, \& Stanley, 2001).

\subsection{L'amplitude articulaire}

Pour ces deux articulations, les procédures sont quasiment identiques. Le dynamomètre isocinétique est programmé pour mobiliser l'articulation jusqu'à $80 \%$ de l'amplitude maximale que le sujet puisse tolérer. Le sujet interrompt la mobilisation en activant le coupe-circuit de sécurité du dynamomètre. Il est donc possible de mesurer simultanément le couple de force passif (généré principalement par les fléchisseurs plantaires ou les ischio-jambiers) et l'angle articulaire, et ceci jusqu'à l'extension passive maximale, à vitesse constante. 


\subsection{La vitesse de mobilisation}

En général, les essais sont réalisés à vitesse angulaire très lente (5 à $10^{\circ} . \mathrm{s}^{-1}$ ), afin d'éviter une réponse réflexe et diminuer la quantité d'énergie absorbée par les tissus étirés (Nordez, McNair, Casari, \& Cornu, 2009).

En effet, les résultats obtenus sur la cheville indiquent que des vitesses angulaires identiques à celles atteintes lors de la marche $\left(25^{\circ} . \mathrm{s}^{-1}\right)$ peuvent être appliquées sans réponse EMG (McNair, Hewson, Dombrowski, \& Stanley, 2002). De même, Hufschmidt et Mauritz (1985) n'ont pas détecté de réponse réflexe lors d'étirement à des vitesses comprises entre 2 et $20^{\circ} \cdot \mathrm{s}^{-1}$, mais seulement à des vitesses supérieures à $80^{\circ} \cdot \mathrm{s}^{-1}$.

\section{La nécessité de l'utilisation de l'électromyographie de surface (EMGs)}

L'utilisation de l'EMGs lors de l'exploration des propriétés passives du SMA est incontournable, car une activité EMG volontaire ou réflexe des muscles étirés modifiera les paramètres caractérisant les propriétés passives du SMA en augmentant par exemple la raideur. Il est donc essentiel de s'assurer que les muscles cibles ne présentent aucune activité lors de l'étirement.

Cet aspect est à considérer par rapport à l'amplitude et à la vitesse du mouvement. Par exemple au niveau de la cheville, la réponse EMGs des muscles fléchisseurs plantaires à l'étirement augmente de manière significative quand ces tissus sont étirés au-delà de $80 \%$ de l'amplitude maximale en dorsiflexion qui peut être mesurée, de façon manuelle 3 à 5 jours avant les tests sur dynamomètre (Elveru, Rothstein, \& Lamb, 1988).

Lors des tests sur dynamomètre, le sujet a pour consigne de se détendre et de ne pas bouger les membres inférieurs. L'activité EMGs des muscles fléchisseurs plantaires et dorsaux de la cheville est contrôlée afin de s'assurer du relâchement du sujet. Les données EMGs et celles issues du dynamomètre sont enregistrées simultanément pour être ensuite traitées. Classiquement, l'activité EMGs est calculée et normalisée par rapport à la contraction maximale volontaire $(\mathrm{CMV})$ mesurée lors de l'expérimentation. L'activité EMGs enregistrée lors des essais peut être ensuite exprimée en pourcentage de l'activation maximale enregistrée lors du test de CMV.

$\mathrm{Si}$ le sujet présente une activité EMGs supérieure à $1 \%$ de la CMV, il n'est pas considéré comme étant relâché, et par conséquent, la mesure n'est pas prise en compte (Gajdosik, et al., 2004; McNair \& Portero, 2005). Pour les sujets âgés (> 60 ans), ce critère étant plus difficile à atteindre, le seuil à partir duquel on peut considérer que le sujet est relâché est situé à $5 \%$ de la CMV (Gajdosik, et al., 2004). Dans certains cas, il est possible d'utiliser un rétrocontrôle visuel de l'EMGs afin de permettre au sujet de visualiser ses activités musculaires et favoriser par conséquent le relâchement. À partir d'observations expérimentales, il apparaît que $5 \%$ des sujets ne

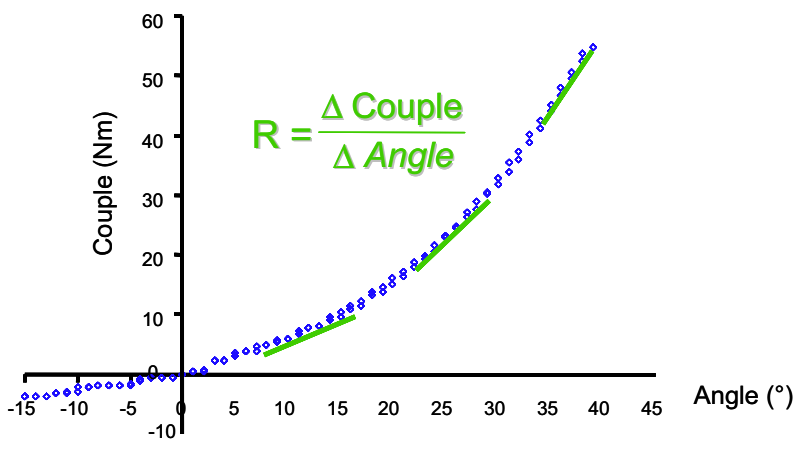

Fig. 2. Relation couple passif - angle articulaire obtenue lors de la mise en tension des muscles postérieurs de la cheville. La raideur du SMA est représentée par la pente de la tangente en chaque point de la relation.

sont pas capables d'être en dessous de ces seuils d'activité EMGs, sans qu'il y ait, apparemment, de pattern d'activation musculaire chez ces sujets. Pour certains, le muscle étiré demeure actif, alors que pour d'autres c'est l'activité des muscles antagonistes qui est perturbante (McNair \& Portero, 2005).

La deuxième raison concerne une vitesse angulaire excessive pouvant entraîner une réponse EMGs. Cependant les essais sont généralement réalisés à vitesse angulaire faible, comme nous l'avons vu précédemment.

En résumé, nous pouvons considérer que les conditions optimales d'exploration des propriétés du SMA en passif sont assurées pour des angles inférieurs à $80 \%$ de l'amplitude articulaire maximale et pour des vitesses d'étirement inférieures à $25^{\circ} . \mathrm{s}^{-1}$, l'activité EMGs étant négligeable pour des sujets sains (Magnusson, et al., 1998; McNair, et al., 2002; McNair \& Portero, 2005).

\section{Les variables mécaniques d'intérêt}

Les variables d'intérêt les plus classiquement obtenues à partir de ces dynamomètres sont le couple résistant, l'angle articulaire et l'énergie passive absorbée (surface sous la courbe). La raideur du SMA mobilisé passivement est calculée par le rapport entre la variation de couple passif et la variation d'angle (Fig. 2).

\subsection{Relation couple passif - angle articulaire}

Une relation non linéaire typique du couple passif en fonction de l'amplitude est obtenue, le couple résistant augmentant précocement mais de façon limitée, puis la pente se redresse (phase linéaire) jusqu'à $80 \%$ de l'amplitude maximale (Fig. 2).

Les études in vitro montrent que durant la phase initiale de la relation, les tissus collagéniques se laissent étirer facilement (grande déformation et faible force) du fait de l'alignement des fibrilles de collagène, puis l'augmentation importante de la force passive est due à la tension et au glissement des fibrilles de collagène entre elles. 


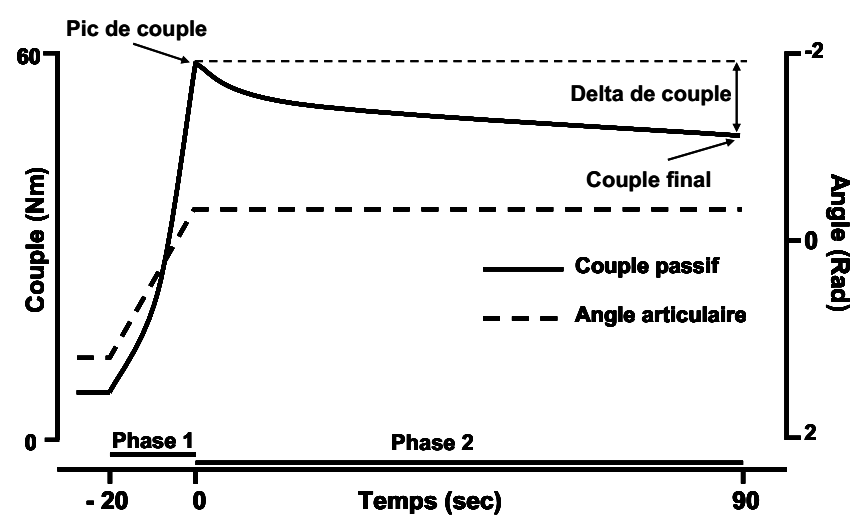

Fig. 3. Réponse mécanique des muscles ischio-jambiers à un étirement statique. Le genou est d'abord mobilisé (phase $1=$ dynamique) à vitesse lente $\left(0,0875 \mathrm{rad} \cdot \mathrm{sec}^{-1}\right.$, soit $\left.5^{\circ} \cdot \mathrm{sec}^{-1}\right)$ jusqu'à une position finale prédéterminée, puis maintenu dans la position (phase $2=$ statique). Le delta de couple (différence entre le pic de couple et le couple final exprimé en $\%$ du pic de couple) caractérise la relaxation de couple viscoélastique (d'après Magnusson, et al., 1995).

Dans de nombreux cas, les chercheurs choisissent une portion particulière de la relation couple-angle pour mesurer la raideur et l'énergie absorbée. Dans certaines études, comme celle de Gajdosik, et al. (2004), le couple a été aussi enregistré lors du retour de l'articulation à la position de départ. De cette façon, ces auteurs ont pu mesurer l'énergie dissipée par le système en calculant la différence entre les surfaces sous la courbe lors de l'étirement et lors du retour, définissant ainsi la notion d'hystérésis qui caractérise le comportement viscoélastique du système.

\subsection{Relaxation de la force}

La relaxation de la force, qui reflète également la viscosité, a été aussi étudiée à l'aide de dynamomètres isocinétiques. Cette méthode consiste à quantifier la diminution du couple passif en fonction du temps, à angle constant (Fig. 3).

Les résultats montrent que la relaxation de la force est décroissante, non linéaire, et asymptotique (McHugh, Magnusson, Gleim, \& Nicholas, 1992; Taylor, Dalton, Seaber, \& Garrett, 1990).

Pour les muscles ischio-jambiers, McHugh, et al. (1992) et Magnusson, et al. (1995) ont enregistré une diminution de 13 à $16 \%$ de la force maximale passive sur une période de 45 secondes, ce qui correspond à nos résultats obtenus sur la cheville, pour laquelle la force diminuait de $17 \%$ sur la même période de temps (McNair, et al., 2001). Cette diminution de couple peut être attribuée à la réponse viscoélastique plutôt qu'à une modification de la réponse contractile détectée par EMGs (Magnusson, et al., 1996). Cette propriété mécanique est associée à la diminution du risque de lésions par étirement (Taylor, et al., 1990).

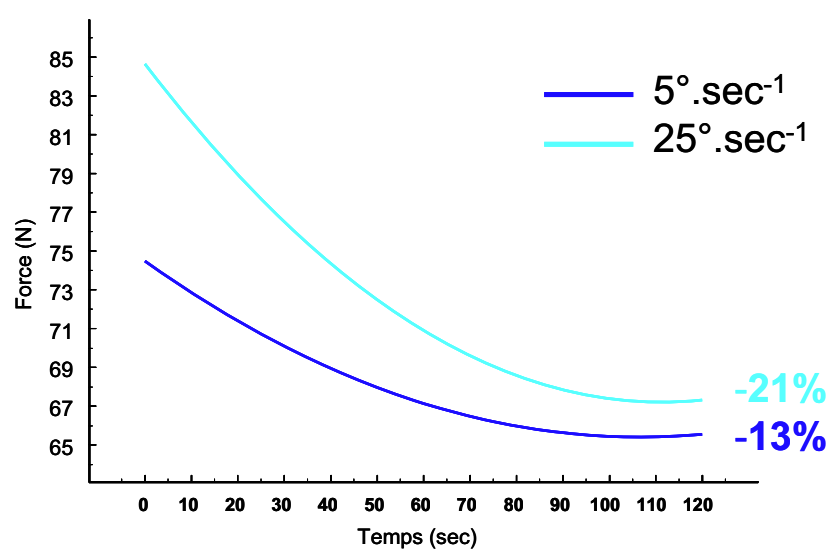

Fig. 4. Modification de la force passive des muscles postérieurs de la cheville en fonction du temps lors d'étirements cycliques pour deux vitesses $\left(5\right.$ et $\left.25^{\circ} \cdot \mathrm{sec}^{-1}\right)$ de mobilisation en dorsiflexion de la cheville (d'après McNair, et al., 2002).

Le même type de réponse est observé lors d'étirements cycliques. En effet, McNair, et al. (2002) ont étudié les effets de mouvements cycliques de dorsiflexion passive de la cheville effectués pendant $2 \mathrm{~min}$. Dans cette expérimentation, réalisée chez des sujets sportifs de loisir, le dynamomètre était programmé pour mobiliser la cheville à une vitesse de $5^{\circ} \cdot \mathrm{s}^{-1}$ sur $80 \%$ de l'amplitude maximale et pendant $120 \mathrm{~s}$. Les résultats ont montré que le pic de force passif de chaque cycle diminuait de façon significative avec le temps. Qualitativement, la tension diminuait de façon curvilinéaire puis s'annulait au bout d'environ $100 \mathrm{~s}$. L'amplitude totale de cette diminution était de $13 \%$. Dans la même étude, ils ont observé les effets de l'augmentation de la vitesse angulaire $\left(25^{\circ} . \mathrm{s}^{-1}\right)$ sur le pic de force passive lors de mouvement cycliques. Leurs résultats montrent que lorsque la vitesse angulaire augmente de $5^{\circ} \cdot \mathrm{s}^{-1}$ à $25^{\circ} \cdot \mathrm{s}^{-1}$, le pic de force passive et la raideur moyenne augmentent également. Sur les $120 \mathrm{mou}-$ vements cycliques, réalisés pour des vitesses angulaires différentes ( 5 et $25^{\circ} . \mathrm{s}^{-1}$ ), la pente de diminution du pic de force passive était plus importante à vitesse rapide (Fig. 4). Ces résultats sont en accord avec ceux de l'étude in vitro de Taylor, et al. (1990), et ceux de l'étude in vivo de Huffschmidt et Schwaller (1987).

\subsection{Fluage}

Le fluage correspond à l'augmentation de la déformation des tissus sous contraintes constantes ou répétées (Thornton, Oliynyk, Frank, \& Shrive, 1997). Le comportement de quasi-fluage peut être mesuré à l'aide de dynamomètre isocinétique. Dans ce domaine sont utilisés des mouvements cycliques pour lesquels le dynamomètre est programmé à inverser le sens du mouvement lorsqu'un niveau déterminé de force ou de couple est atteint. Une étude pilote (citée par McNair \& Portero, 2005) a montré que les sujets peuvent accepter un niveau de force de $85 \%$ de la force maximale mesurée lors d'un test de tolérance 
à l'étirement sans inconfort-douleur. L'extension maximale était le paramètre recherché. Les résultats initiaux montrent que cette variable peut augmenter de 4,5\% au cours des 45 cycles effectués, la plus grande augmentation s'observant dans les 10 à 15 premiers cycles.

\subsection{Association de l'échographie aux mesures dynamométriques}

Le couplage de l'échographie aux dynamomètres isocinétiques lors de tests passifs ont permis récemment de quantifier les contributions des tissus tendineux (tendon et aponévrose) et des fascicules musculaires à l'allongement du système musculo-tendineux (Herbert, Moseley, Butler et Gandevia, 2002; Herbert, et al., 2011; Abellaneda, Guissard, \& Duchateau, 2009). L'échographie en temps réel donne la possibilité de mesurer le déplacement de la jonction myotendineuse et l'élongation des fascicules musculaires pour en déduire la modification de longueur des tissus tendineux et musculaires lors du mouvement passif de l'articulation (Herbert, et al., 2002; Muraoka, Muramatsu, Takeshita, Kawakami, \& Fukunaga, 2002). Il est alors possible de passer d'une relation couple passif-angle à une relation couple-allongement. Herbert, et al. $(2002,2011)$ ont ainsi montré qu'entre $50 \%$ (jambe tendue) et $73 \%$ (jambe fléchie) de l'allongement passif des gastrocnemii est dû à l'étirement des tissus tendineux. L'allongement du tendon contribue donc significativement au comportement mécanique du système muscle-tendon en passif bien que sa raideur soit supérieure à la raideur musculaire en passif. Ce résultat s'explique principalement par une longueur du tendon plus importante que la longueur des fascicules musculaires.

Afin d'évaluer plus précisément les propriétés mécaniques des structures musculo-tendineuses, Hoang, Gorman, Todd, Gandevia, \& Herbert (2005) ont proposé une méthode permettant d'estimer la relation forcelongueur passive du système muscle-tendon à partir de relations couple-angle obtenus lors d'étirements passifs de la cheville en flexion dorsale à différents angles du genou. En combinant cette méthode avec une mesure échographique, les relations force-longueur du muscle et du tendon peuvent également être déterminées (Hoang, Herbert, Todd, Gorman, \& Gandevia, 2007). Enfin, en mesurant les sections anatomiques musculaires et tendineuses, il devient possible d'établir les relations contrainte-déformation du muscle et du tendon.

\section{Conclusion}

Les mesures spécifiques du couple passif, de l'amplitude articulaire, et le calcul de la raideur et de l'absorption d'énergie passive peuvent être réalisés à l'aide de dynamomètres isocinétiques et permettent de caractériser le SMA en mode passif. Par ailleurs, les variations de ces paramètres peuvent être enregistrées en fonction du temps, et permettent ainsi d'approcher les notions de relaxation de force ou de fluage et donc évaluer les propriétés viscoélastique du SMA.

De nombreux travaux, utilisant ces dynamomètres isocinétiques et d'autres outils comme l'échographie, ont contribué à l'amélioration des connaissances théoriques sur le comportement du SMA, sur ses adaptations à court terme et à long terme (e.g. stretching, conditionnement et déconditionnement musculaire), et à préciser les recommandations spécifiques au stretching.

\section{Bibliographie}

Abellaneda, S., Guissard, N., \& Duchateau, J. (2009). The relative lengthening of the myotendinous structures in the medial gastrocnemius during passive stretching differs among individuals. Journal of Applied Physiology, 106, 169-177.

Damiano, D.L., Quinlivan, J.M., Owen, B.F., Payne, P., Nelson, K.C., \& Abel, M.F. (2002). What does the Ashworth scale really measure and are instrumented measures more valid and precise? Developmental Medicine \& Child Neurology, 44, 112-118.

Elveru, R., Rothstein, J., \& Lamb, R. (1988). Goniometric reliability in a clinical setting. Subtalar and ankle joint measurements. Physical Therapy, 68, 672-677.

Engsberg, J.R., Olree, K.S., Ross, S.A., \& Park, T.S. (1996). Quantitative clinical measure of spasticity in children with cerebral palsy. Archives of Physical Medicine \& Rehabilitation, 77, 594-599.

Engsberg, J.R., Ross, S.A., Olree, K.S., \& Park, T.S. (2000). Ankle spasticity and strength in children with spastic diplegic cerebral palsy. Developmental Medicine \& Child Neurology, 42, 42-47.

Farcy, S., Portero, P., \& Rabita, G. (2011). Compliance de la composante élastique série : structures physiologiques impliquées et méthodes d'évaluation. In : M. Julia, D. Hirt, J-L. Croisier, \& P. Codine (Eds). Tendon et jonction tendinomusculaire - De la biomécanique aux applications thérapeutiques (pp. 15-23). Paris: Masson.

Gajdosik, R.L. (2001). Passive extensibility of skeletal muscle: review of the literature with clinical implications. Clinical Biomechanics, 16, 87-101.

Gajdosik, R.L., Vander-Linden, D.W., McNair, P.J., Riggin, T.J., Albertson, J.S., Mattick, D.J., \& Wegley, J.C. (2004). Slow passive stretch and release characteristics of the calf muscles of older women with limited dorsiflexion range of motion. Clinical Biomechanics, 19, 398-406.

Gajdosik, R.L., Vander-Linden, D.W., McNair, P.J., Riggin, T.J., Albertson J.S., Mattick, D.J., \& Wegley, J.C. (2005). Viscoelastic properties of short calf muscle-tendon units of older women: effects of slow and fast passive dorsiflexion stretches in vivo. European Journal of Applied Physiology, 95, 131-139.

Goubel, F., \& Lensel-Corbeil, G. (2003). Biomécanique. Eléments de mécanique musculaire. Paris: Masson. 
Halbertsma, J.P.K., Mulder, I., Goeken, L.N.H., Gijeken, L.N.H., \& Eisma, W.H. (1999). Repeated passive stretching: acute effect on the passive muscle moment and extensibility of short hamstrings. Archives of Physical Medicine \& Rehabilitation, 80, 407-414.

Herbert, R.D., Clarke, J., Kwah, L.K., Diong, J., Martin, J., Clarke, E.C., Bilston, L.E., \& Gandevia, S.C. (2011). In vivo passive mechanical behaviour of muscle fascicles and tendons in human gastrocnemius muscle-tendon units. Journal of Physiology, 589, 5257-5267.

Herbert, R.D., Moseley, A.M., Butler, J.E., \& Gandevia, S.C. (2002). Change in length of relaxed muscle fascicles and tendons with knee and ankle movement in humans. Journal of Physiology (London), 539, 637-645.

Hill, A.V. (1951). The effect of series compliance on the tension developed in muscle twitch. Proceedings of the Royal Society B: Biological Sciences, 138, 325-329.

Hoang, P.D., Gorman, R.B., Todd, G., Gandevia, S.C. \& Herbert, R.D. (2005). A new method for measuring passive length-tension properties of human gastrocnemius muscle in vivo. Journal of Biomechanics, 38, 1333-1341.

Hoang, P.D., Herbert, R.D., Todd, G., Gorman, R.B. \& Gandevia, S.C. (2007). Passive mechanical properties of human gastrocnemius muscle tendon units, muscle fascicles and tendons in vivo. Journal of Experimental Biology, 210, 4159-4168.

Hufschmidt, A., \& Mauritz, K. (1985). Chronic transformation of muscle in spasticity: a peripheral contribution to increased tone. Journal of Neurology, Neurosurgery \& Psychiatry, 48, 676-685.

Hufschmidt, A., \& Schwaller, I. (1987). Short range elasticity and resting tension of relaxed human lower leg muscles. Journal of Physiology (London), 391, 451-465.

Lamontagne, A., Malouin, F., \& Richards, C. (1997). Viscoelastic behaviour of the plantar flexor muscle tendon unit at rest. Journal of Orthopaedic \& Sports Physical Therapy, 26, 244-252.

Magnusson, P., Aagard, P., Simonsen, E., \& Bojsen-Moller, F. (1998). A biomechanical evaluation of cyclic and static stretch in human skeletal muscle. International Journal of Sports Medicine, 19, 310-316.

Magnusson, P., Simonsen, E., Aagaard, P., Gleim, GW., McHugh, MP., \& Kjaer, M. (1995). Viscoelastic response to repeated static stretching in the human hamstring muscle. Scandinavian Journal of Medicine \& Science in Sports, 5, 342-347.

Magnusson, P., Simonsen, E., Dyhre-Poulsen, P., Aagaard, P., Mohr, T., \& Kjaer, M. (1996). Viscoelastic stress relaxation during static stretch in human skeletal muscle in the absence of EMG activity. Scandinavian Journal of Medicine \& Science in Sports, 6, 323-328.

Maïsetti, O., Sastre, J., Lecompte, J., \& Portero, P. (2007). Effects of 5-repeated static stretches on rate of voluntary force development and maximal strength of ankle plantar flexor muscles. Isokinetics \& Exercise Science, 15, 11-17.

McHugh, M., Magnusson, P., Gleim, G., Nicholas, J. (1992). Viscoelastic stress relaxation in human skeletal muscle. Medicine \& Science in Sports \& Exercise, 24, 1375-1382.

McNair, P., Dombroski, E., Hewson, D., \& Stanley, S. (2001). Stretching at the ankle joint: viscoelastic responses to holds and continuous passive motion. Medicine \& Science in Sports \& Exercise, 33, 354-358.

McNair, P., Hewson, D., Dombroski, E., \& Stanley, S. (2002). Stiffness and passive peak force changes at the ankle joint: the effect of different joint angular velocities. Clinical Biomechanics, 17, 536-540.

McNair, P., \& Portero, P. (2005). Using isokinetic dynamometers for measurements associated with tissue extensibility. Isokinetics \& Exercise Science, 13, 53-56.

Muraoka, T., Muramatsu, T., Takeshita, D., Kawakami, Y., \& Fukunaga, T. (2002). Length change of human gastrocnemius aponeurosis and tendon during passive joint motion. Cells Tissues Organs, 171, 260-268.

Nordez, A., Cornu, C., \& McNair, P.J. (2006). Acute effects of static stretching on passive stiffness of the hamstring muscles calculated using different mathematical models. Clinical Biomechanics, 21, 755-760.

Nordez, A., Casari, P., \& Cornu, C. (2008). Accuracy of Biodex system 3 pro computerized dynamometer in passive mode. Medical Engineering \& Physics, 30, 880-887.

Nordez, A., McNair, P.J., Casari, P., \& Cornu, C. (2009). The effect of angular velocity and cycle on the dissipative properties of the knee during passive cyclic stretching: A matter of viscosity or solid friction. Clinical Biomechanics, 24, $77-81$.

Pincivero, D.M., Lephart, S.M., \& Karunakara, R.A. (1997). Reliability and precision of isokinetic strength and muscular endurance for the quadriceps and hamstrings. International Journal of Sports Medicine, 18, 113-117.

Rabita, G., Dupont, L., Thevenon, A., Lensel-Corbeil, G., Pérot, C., \& Vanvelcenaher, J. (2005). Quantitative assessment of the velocity-dependent increase in resistance to passive stretch in spastic plantarflexors. Clinical Biomechanics, 20, 745-753.

Ross, S.A., \& Engsberg, J.R. (2002). Relation between spasticity and strength in individuals with spastic diplegic cerebral palsy. Developmental Medicine \& Child Neurology, 44, 148-157.

Silder, A., Heiderscheit, B., \& Thelen, D.G. (2008). Active and passive contributions to joint kinetics during walking in older adults. Journal of Biomechanics, 41, 1520-1527.

Smith, C. (1994). The warm up procedure: to stretch or not to stretch. A brief review. Journal of Orthopaedic \& Sports Physical Therapy, 19, 12-17.

Taylor, D., Sanders, R.H., Howick, E.I., Stanley, S.N. (1991). Static and dynamic assessment of the Biodex dynamometer. European Journal of Applied Physiology, 62, 180-188.

Taylor, D., Dalton, J., Seaber, A., \& Garrett, W. (1990). Viscoelastic properties of muscle-tendon units. The biomechanical effects of stretching. American Journal of Sports Medicine, 18, 300-309.

Thornton, G., Oliynyk, A., Frank, C., \& Shrive, N. (1997). Ligament creep cannot be predicted from force relaxation at low stress: a biomechanical study of the rabbit medial collateral ligament. Journal of Orthopaedic Research, 15, 652-656.

Zajac, F.E. (1989). Muscle and tendon: properties, models, scaling, and application to biomechanics and motor control. Critical Reviews in Biomedical Engineering, 17, $359-411$. 